\title{
Boron neutron capture therapy for newly diagnosed glioblastoma
}

Tetsuya Yamamoto MD, PhD, Kei Nakai MD, PhD, Teruyoshi Kageji MD, $\mathrm{PhD}^{\dagger}$, Hiroaki Kumada $\mathrm{PhD}^{\dagger \dagger}$, Kiyoshi Endo MD, PhD, Masahide Matsuda, MD, Yasushi Shibata MD, $\mathrm{PhD}$, Akira Matsumura MD, PhD.

\section{Affiliation:}

Department of Neurosurgery, Institute of Clinical Medicine, University of Tsukuba ${ }^{\dagger}$ Department of Neurosurgery, Tokushima University $\dagger$

${ }^{\dagger \dagger}$ Department of Research Reactor and Tandem Accelerator, Japan Atomic Energy Agency

Correspondence and reprint requests to:

Tetsuya Yamamoto, M.D., Ph.D.

Department of Neurosurgery, Institute of Clinical Medicine, University of Tsukuba

Tennnodai 1-1-1, Tsukuba City, Ibaraki, 305-8575, Japan

Email: tetsu-ya@md.tsukuba.ac.jp

The total number of pages: 13

Running head: neutron capture therapy for glioblastoma

Key words: glioblastoma, radiation, neutron capture therapy 


\begin{abstract}
Purpose: The efficacy, safety, and dose distribution of neutron capture therapy (NCT) were evaluated in 15 patients with newly diagnosed glioblastoma.

Methods and materials: Seven patients received intraoperative NCT (protocol-1) and 8 patients received external beam NCT (protocol-2). Sulfhydryl borane (5g/body) was administered intravenously. Additionally, $p$-dihydroxyboryl-phenylalanine $(250 \mathrm{mg} / \mathrm{kg})$ was given in protocol-2. The external beam NCT was combined with fractionated photon irradiation.

Results: Four of 15 patients were alive at analysis for a mean follow-up time from diagnosis of $23.0 \mathrm{M}$. Twelve of the 15 patients followed up for more than one year, and 10 (83.3\%) of the 12 patients maintained their Karnofsky Performance Status (KPS; 90 in 8 and 100 in 2) at 12 months. The median overall survival and the time to tumor progression (TTP) for all patients were $25.7 \mathrm{M}$ and $11.9 \mathrm{M}$, respectively. There was no difference in TTP between the protocol-1 (12.0 M) and protocol-2 (11.9 M). The 1- and 2-year survival rates were $80.0 \%$ and $53.3 \%$, respectively. Three protocol-1 patients and one protocol-2 patient suffered transient orbital swelling accompanied by double vision (Grade 2); 1 of the 3 protocol-1 patients suffered post-epileptic brain swelling (Grade 4) requiring surgical intervention.

Conclusion: It is suggested that NCT is effective for survival of newly-diagnosed glioblastoma with acceptable adverse effects. Because of the limitation of the present NCT pilot study without the contemporary control arm, whether the neutron capture reaction led to distinct survival benefits is unconvincing and further optimized studies on less invasive external beam NCT in large series of patients are warranted.
\end{abstract}




\section{Introduction}

Glioblastoma multiforme (GBM) is one of the most frequent primary brain tumors in adults. Despite recent advancements in image-guided neurosurgery, conformal radiation and chemotherapeutic agents, GBM is still an incurable disease that recurs easily due to rapid growth and microscopic invasion to the surrounding normal brain tissue. Concomitant and adjuvant use of temozolomide with conventional photon radiotherapy, the new standard post-operative therapy for GBM, has demonstrated a significant survival advantage compared to radiotherapy alone, and shows minimal additional toxicity. The median overall survival (OS) time in a randomized control trial was $14.6 \mathrm{M}$ with temozolomide plus radiotherapy and $12.1 \mathrm{M}$ with radiotherapy alone [1].

The dose of radiation required to completely kill GBM cells has not yet been determined, however, previous reports imply that at least 90 Gy must be used to achieve better local control $[2,3]$. In general, such high-dose radiation is considered to be in excess of the tolerance dose of normal brain tissue which is used in conventional fractionated photon radiotherapy. Several randomized trials have shown a survival benefit of conventional fractionated photon radiotherapy at total doses of 45 to $60 \mathrm{~Gy}$; the median OS in these trials was $5.8 \mathrm{M}$ to $15.5 \mathrm{M}$ [4-7]. Most subsequent dose escalation studies have been designed as case series of small numbers of selected patients who underwent additional stereotactic radiosurgery, fractionated proton beam radiation or other conformal radiotherapy. The better outcome following these radiotherapies, in which median OS varies from $9.5 \mathrm{M}$ to $25 \mathrm{M}$, is in part the result of patient selection $[2,3,8-10]$. There is an urgent need for a tumor-selective high-dose therapy that would encompass the main tumor mass as well as microscopic invasion whilst avoiding radiation damage to the surrounding normal brain tissue.

Neutron capture therapy (NCT) preferentially destroys ${ }^{10} \mathrm{~B}$-loaded tumor cells, while sparing the normal tissue without ${ }^{10} \mathrm{~B}$, based on a nuclear reaction between ${ }^{10} \mathrm{~B}$, a stable non-radioactive isotope of boron, and low energy thermal neutrons, which release high linear energy transfer (LET) $\alpha\left({ }^{4} \mathrm{He}\right)$ particles and ${ }^{7} \mathrm{Li}$ particles with a path-length of one cell diameter $(-10 \mu \mathrm{m})$ through the boron neutron capture reaction, ${ }^{10} \mathrm{~B}(\mathrm{n}, \alpha){ }^{7} \mathrm{Li}$. Therefore, NCT theoretically allows a unique tumor-cell-selective radiotherapy existing of all radiation therapy modalities. The effectiveness of NCT is highly dependent on the optimization of the accumulation of selective ${ }^{10} \mathrm{~B}$-containing agent(s) in tumor cells as well as on the presence of sufficient thermal neutrons even at the deepest levels. Although there have been a great number of pre-clinical and clinical investigations over the last six decades [11-30,35-42], this emerging therapeutic modality remains immature compared to 
recent systematically computerized and accurate conformal radiation modalities. There are some issues that remain to be resolved, given that neutron sources are restricted to nuclear reactors, limiting their availability for conformal neutron beam irradiation and more efficient ${ }^{10} \mathrm{~B}$-containing agents.

Recent clinical studies on boron NCT (BNCT) have focused on high-grade glioma and malignant meningioma [11-23], head and neck tumors [24,29], cutaneous melanoma $[25,26]$ and lung and liver metastases $[27,28]$ as potential candidates for NCT. To the best of our knowledge, no clinical data have yet been published on NCT in a randomized controlled trial. With respect to the case series as Phase I or Phase II NCT trials for GBM, the median OS time varied from $13 \mathrm{M}$ to $20.7 \mathrm{M}$. Although these values are similar to those of postoperative conventional photon fractionation and standard chemotherapy [1], they imply that NCT may be at least partially effective. In the present pilot study, survival benefits, safety and dose distribution in tumor and normal tissue were evaluated in GBM patients who were treated with either the former intra-operative NCT (IO-NCT) or the current less-invasive external beam NCT (EB-NCT).

\section{Materials and Methods}

As boron delivery agents for $\mathrm{NCT}$, sulfhydryl borane $\mathrm{Na}_{2} \mathrm{~B}_{12} \mathrm{H}_{11} \mathrm{SH}$ (BSH) and p-dihydroxyboryl-phenylalanine (BPA) were used in this study. BSH (5 g/body, 1 hour i.v. infusion) was administered intravenously 12 hours before neutron irradiation in IO-NCT (protocol-1) and EB-NCT (protocol-2). Additionally, water soluble fructose complex of BPA $(250 \mathrm{mg} / \mathrm{kg}, 1$ hour i.v. infusion) was given 1.5 hour before irradiation for the 8 patients who underwent EB-NCT. Blood samples were drawn serially after intravenous injection to estimate the blood boron level. The blood samples were measured by inductively coupled plasma-atomic emission spectroscopy (ICP-AES) and confirmed by prompt gamma ray analysis (PGA). A tumor-to-BSH-mediated blood ${ }^{10} \mathrm{~B}$ concentration ratio of 1.0 was used to estimate the boron dose to be delivered to the tumor tissue.

${ }^{18} \mathrm{~F}$-labeled BPA positron emission tomography (PET) was performed prior to EB-NCT in order to calculate the lesion-to-normal ratio of BPA-mediated ${ }^{10} \mathrm{~B}$ for estimating the BPA-mediated tumor dose in EB-NCT

Neutron irradiation in both protocol-1 and protocol-2 was performed in a single fraction using an epithermal or epithermal-thermal mixed beam of Japan Research Reactor No. 4 (JRR-4). The beam characteristics have been described elsewhere [30]. Briefly, the thermal $(<0.53 \mathrm{eV})$, epithermal $(0.53 \mathrm{eV}-10 \mathrm{keV})$ and fast $(>10 \mathrm{keV})$ neutron flux and gamma ray absorbed dose for the epithermal beam were $3.6 \times 10^{8} \mathrm{n} \mathrm{cm}^{-2} \mathrm{~s}^{-1}, 2.2 \times 10^{9} \mathrm{n}$ $\mathrm{cm}^{-2} \mathrm{~s}^{-1}, 1.1 \times 10^{8} \mathrm{n} \mathrm{cm}^{-2} \mathrm{~s}^{-1}$ and $2.4 \mathrm{~Sv} / \mathrm{h}$, respectively, at a reactor power of $3.5 \mathrm{MW}$. The 
thermal, epithermal and fast neutron flux and gamma ray absorbed dose for the epithermal-thermal beam were $2.0 \times 10^{9} \mathrm{n} \mathrm{cm}^{-2} \mathrm{~s}^{-1}, 9.0 \times 10^{8} \mathrm{n} \mathrm{cm}^{-2} \mathrm{~s}^{-1}, 3.6 \times 10^{5} \mathrm{n} \mathrm{cm}^{-2} \mathrm{~s}^{-1}$ and $3.6 \mathrm{~Sv} / \mathrm{h}$, respectively. Additional photon irradiation, at a total X-ray dose of $30 \mathrm{~Gy}$ in 15 fractions or $30.6 \mathrm{~Gy}$ in 17 fractions, was given to high-intensity areas on T2-weighted magnetic resonance (MR) images to compensate for insufficiency of the dose distribution in protocol-2 relative to protocol-1 (Fig. 1).

The photon-equivalent BNCT dose was calculated by multiplied the weighting factor to account for the increased biological effectiveness of the high-LET dose components.; $a$ and ${ }^{7} \mathrm{Li}$ particles through the boron neutron capture reaction, ${ }^{10} \mathrm{~B}(\mathrm{n}, \alpha){ }^{7} \mathrm{Li}$, the proton recoils due to fast neutron, ${ }^{1} \mathrm{H}(\mathrm{n}, \mathrm{n}) \mathrm{p}, 0.54 \mathrm{MeV}$ protons from the nitrogen capture reaction, ${ }^{14} \mathrm{~N}(\mathrm{n}, \mathrm{p}){ }^{14} \mathrm{C}[20,30]$. The maximum normal brain point $(5 \mathrm{x}$ $5 \times 5 \mathrm{~mm}$ voxel) dose prescribed was limited to 13.0 Gy or less. The dose estimation for protocol-1, which was carried out by the gold wire activation method using a thermoluminescent dosimeter, was validated by the BNCT dose simulation software, Japan Atmic Energy Agency (JAEA) Computational Dosimetry System (JCDS). For protocol-2, the various prescribed doses, including the minimum tumor dose at clinical target volume (CTV)-1, CTV-2 and CTV-3, the maximum normal brain dose, the maximum skin dose and the average brain dose were calculated using JCDS. CTV-1 was defined as gross residual or unreseced tumor which was shown as Gd-enhanced area in MR images within three days after surgery. In a case without Gd-enhanced area, 5-mm-margin of post-surgical cavity was defined as CTV-1. CTV-2 (3) was defined as the volume which consists of 2 (1)-cm around CTV-1 (CTV-2).

The BNCT protocols in the present study were approved by the Medical Ethics Committee of the University of Tsukuba, and all participating patients were fully informed and provided their written informed consent. Following the 2005 policy of the International Committee of Medical Journal Editors (ICMJE), the later stage of this trial (protocol-2) was registered with the Japanese authority on clinical trial registration (University Hospital Medical Information Network Clinical Trial Registry: UMIN-CTR; Trial ID: C000000298). The clinical trial was closed because of neutron beam availability at JRR-4 which has been reconstructed for the improvement of a reactor core.

Between 1998 and 2007, we treated 71 patients aged 31 to 84 years (median, 60) with newly diagnosed, histologically confirmed GBM. Eligibility of the patients was decided by more than three neurosurgeons and two radiotherapists. Fifteen eligible patients with signed informed consent who underwent maximal safe resection were selected as participants in the present study (protocol-1, $\mathrm{n}=7$; protocol-2, $\mathrm{n}=8$ ); Supratentorial unilateral tumors located no deeper than $7 \mathrm{~cm}$ from the brain surface, with a Karnofsky 
Performance Status (KPS) of 50 or more, who had undergone no previous chemotherapy or radiotherapy and no previous therapy for any other cancers, and who had no allergy to BSH or BPA. None of the patients in this series had a previous malignancy.

The patients' characteristics are shown in Table 1. The median age at primary diagnosis was 57 years (range, 32-76). All patients underwent surgical removal before BNCT, which consisted of gross total removal in 4 (26.7\%), partial removal in 10 (66.7\%) and biopsy in $1(6.6 \%)$. Five of the 7 protocol-1 patients were described previously in an interim report [11]. The median age of the protocol-1 patients was 51 years (range, 38-64) and that of the protocol-2 patients was 65 years (range, 32-76). The median interval between surgery and NCT was 40 days (range, 29-95) in the protocol-1 group and 28 days (range, 9-49) in the protocol-2. Two of 15 patients (13.3\%) were categorized in the best prognostic group of GBM (class III) by the Radiation Therapy Oncology Group (RTOG) Recursive Partitioning Analysis (RPA) as were 3 of 15 patients (20\%) by the European Organization for Research and Treatment of Cancer (EORTC) RPA [31,32].

All patients were followed up clinically and by MR imaging every 1-3 M. The time to tumor progression (TTP) was determined by appearance or enlargement of Gd-enhancement on post-BNCT MR image, in this study. Both OS and TTP were defined as the interval from the primary diagnosis. Adverse events were graded according to the National Cancer Institute (NCI) Common Toxicity Criteria, version 2 [33].

Survival time and survival rate were estimated using the Kaplan-Meier method to assess the efficacy of NCT. The log-rank test was used to compare survival times in each type of NCT. These statistical analyses were performed using SPSS, version 15.0J (SPSS Japan, Inc., Tokyo, Japan).

\section{Results}

The blood boron concentration at the time of neutron irradiation averaged $32.9 \pm 12.2$ $\mu \mathrm{g} / \mathrm{g}$ for $\mathrm{BSH}$ in protocol-1, and $34.6 \pm 9.6 \mu \mathrm{g} / \mathrm{g}$ for $\mathrm{BSH}$ and $17.4 \pm 2.4 \mu \mathrm{g} / \mathrm{g}$ for BPA in protocol-2. The maximum normal brain doses for protocol-1 and protocol-2 were $29.4 \pm 6.0$ Gy (range, 20.1-36.0) and 11.4 \pm 1.5 Gy (range, 8.4-14.1), respectively. In protocol-2, the minimum tumor doses of CTV-1, CTV-2 and CTV-3 averaged 29.8 +9.9 Gy, 15.1 \pm 5.4 Gy and 12.4 $\pm 2.9 \mathrm{~Gy}$, respectively. The maximum brain dose, skin dose and average brain dose were 11.4 $\pm 1.5 \mathrm{~Gy}, 9.5 \pm 1.5 \mathrm{~Gy}$ and $3.9 \pm 1.8 \mathrm{~Gy}$, respectively. The average brain doses in protocol-1 and protocol-2 were $5.1 \pm 2.2$ Gy and 3.1 \pm 0.4 Gy, respectively.

The most common acute adverse event was mild erythema (Grade 1), which was observed in most patients. Three protocol-1 patients and 1 protocol-2 patient suffered transient orbital swelling accompanied by double vision (Grade 2). Three protocol-1 
patients received frontotemporal irradiation without ideal neutron shielding due to skin reflection. One of these three patients suffered post-epileptic brain swelling (Grade 4) requiring surgical intervention; this patient died of pneumonia 14.1 M after NCT. No serious BSH- or BPA-related toxicity was observed in the present series. Low-grade fever (Grade 1), vasoconstruction (Grade 1), and erythema, itching or vascular pain following intravenous infusion of BSH (Grade 1) were observed in 5 cases during protocol-1.

Four of 15 patients were still alive at analysis for a mean follow-up time of $23.0 \mathrm{M}$. Twelve of the 15 patients followed up for more than one year, and $10(83.3 \%)$ of the 12 patients maintained their Karnofsky Performance Status (KPS; 90 in 8 and 100 in 2) at 12 months. The median OS and median TTP for all patients were $25.7 \mathrm{M}$ and $11.9 \mathrm{M}$, respectively. There was no significant difference in median OS and TTP between the protocol-1 (OS: 23.3 M, TTP: 12.0 M) and protocol-2 (OS: 27.1 M, TTP: 11.9 M) groups, respectively (Fig. 2A, 2B). The 1- and 2-year survival rates were $80.0 \%$ and $53.3 \%$, respectively. Treatment of tumor progression included neurosurgical resection $(n=7)$ and chemotherapy $(\mathrm{n}=5)$, and no patient underwent a second course of radiation.

\section{Discussion}

Postoperative NCT following debulking surgery in 15 selected patients with newly diagnosed GBM was found to compare favorably with other series of conventional or high-dose radiotherapies [1-3]. No serious adverse events were observed in the patients who had better neutron shielding.

The results of the present study could not be directly compared to those of historical controls or to those previously reported in a number of publications because of the present small case series of selected patients, which was a major limitation. However, the prognostic factor-based classifications utilized in the comparison of similar small cohorts $[2,31,32]$, suggest that these relatively positive data are unlikely to reflect patient selection alone. In a subanalysis of the EORTC 26981/22981-NCIC CE3 phase III randomized trial on conventional radiotherapy and temozolomide for newly diagnosed GBM, the median OSs in EORTC-RPA Classes III, IV and V were 17, 15 and $10 \mathrm{M}$, respectively [32]. The most favorable 2-year rate (43.4\%) was also scored in Class III. Curran et al. report that the OS after surgery and several radiation regimens using 60 Gy to 81.6 Gy for 1578 patients entered in three RTOG trials were categorized into four (III to VI) of 6 classes with median OSs for Classes III, IV, V and VI of 17.9, 11.1, 8.9 and 4.6M, respectively. The most favorable 2-year rate (35\%) was also scored in Class III. Moreover, in high-dose radiation therapy using a proton beam, RTOG prognostic Classes III, IV and V had median OSs of 23, 17 and $14 \mathrm{M}$, respectively, and Class III had a 2 -year survival rate of $34 \%$ [2]. In the present series, only 2 of 15 patients (13.3\%) were 
categorized as RTOG Class III and 3 of 15 (20\%) were categorized as EORTC Class III. It is noteworthy that the median OS $(25.7 \mathrm{M})$ and 2-year survival rate $(53.3 \%)$ in the present series appear to be superior to those of RTOG Class III and EORTC Class III in both standard therapy and reported high-dose therapy. In a previous nonrandomized trial of high-dose radiation with radiosurgery boost, reoperation was performed in half of the patients. Histopathological diagnosis showed half of these had radiation necrosis [33]. Reoperation has been shown to be a positive prognostic factor in the patients with GBM who underwent high-dose radiotherapy $[2,33]$. In this series, 7 patients with post-NCT deterioration on MR images suffered neurological deterioration with minimal performance decline, and had a subsequent resection. Pathological diagnosis was recurrent tumor in 2 (case 6,8), whereas radiation necrosis with or without tumor cells in 5 (case 7,12,13,14,15).

Hatanaka and Nakagawa treated more than 200 patients with high-grade glioma with BSH-mediated IO-NCT, achieving a median OS of 22.9 M for GBM [35]. We previously reported 9 patients (GBM in 5, anaplastic astrocytomas in 4) with high-grade gliomas who were treated with BSH-mediated IO-NCT using a mixed epithermal beam of JRR-4 [11]. The median OS for the $5 \mathrm{GBM}$ patients was $23.2 \mathrm{M}$. IO-NCT under 2 different protocols reported by Kageji [14] showed median OSs for higher and lower dose groups of 19.5 and $15.3 \mathrm{M}$, respectively. In the present series, additional photon fractionation and the combination of two boron agents were employed in EB-NCT, resulting in at least identical 2-year survival rates in EB-NCT (66.7\%), compared to IO-NCT (42.9\%). The use of additional photon radiation was based on the experimental data of Barth et al., which showed that the combination of BNCT and photon irradiation could enhance therapeutic effects in tumor-bearing rats [36]. BSH biodistribution studies have suggested that the primary mode of selective BSH distribution is passive diffusion from blood to tumor tissue via the disrupted blood brain barrier (BBB) [37,38]. The combination of BPA and BSH $[12,39]$ as well as a longer infusion of BPA $[18,40,41]$ appear to minimize the heterogeneous ${ }^{10} \mathrm{~B}$ distribution in both experimental and clinical situations.

It has been reported that the majority of central nervous system toxicities in NCT are acute, self-limiting and primarily related to a temporal increase in intracranial pressure [20]. A tumor volume of more than $60 \mathrm{~cm}^{3}$, an increased average brain dose, and an increased number of irradiation fields were found to lead to a higher incidence of such toxicities. An average brain dose of 6.2 Gy-Eq was associated with a $50 \%$ incidence of somnolence. As a consequence of the smaller tumor volume of $18.6 \pm 12.2 \mathrm{~cm}^{3}$ and rather localized dose planning, lower average brain doses $(3.9 \pm 1.8 \mathrm{~Gy})$ were used in the present series compared to previously reported doses. The higher than average brain dose in the IO-NCT group $(5.1 \pm 2.2 \mathrm{~Gy})$ compared to the EB-NCT group $(3.1 \pm 0.4 \mathrm{~Gy})$ may have contributed to the higher incidence of toxicities.

In conclusion, although NCT is the most complicated radiotherapy, it may be a valuable 
future alternative treatment for cancer that invades the surrounding healthy normal tissue, and may be preferable to currently existing high-conformal radiation therapies, since in theory it allows tumor-cell-selective high-dose radiation while sparing normal cells. Because of the limitations of the present NCT pilot study for the newly diagnosed GBM without the contemporary control arm, it is not convincing whether the neutron capture reaction is functioning enough to control tumors and led to distinct survival benefits. Randomized trials of comparably selected patients are required to demonstrate conclusively that prolonged survival is a result of this tumor-selective radiotherapy. It is suggested that post-operative EB-NCT is effective for newly-diagnosed glioblastoma and is less invasive than IO-NCT. Future optimization of tumor-targeting boron agents and their delivery, combination with other modalities, and the development of an in-hospital accelerator-based neutron source will be required for enhancing the therapeutic effectiveness of BNCT [13].

\section{Acknowledgments}

This study was supported in part by a Grant-in-Aid for Society Collaboration from the Ministry of Education, Science and Culture, Japan (17390390). We thank Dr. Yoshinobu Nakagawa (IO-NCT procedure), Dr. Koji Ono (EB-NCT procedure), Dr. Shin-Ichi Miyatake (EB-NCT procedure), Dr. Koji Tsuboi (Photon irradiation), Dr. Koichi Tokuue (Photon irradiation), Dr. Alexander Zaboronok (manuscript preparation), and Dr. Tadashi Nariai $\left({ }^{18}\right.$ F-BPA-PET) for their kind support. T. Y., K. N., A. M. contributed equally in this study as primary co-investigators.

\section{References}

1. Stupp R, Mason WP, van den Bent MJ, et al. Radiotherapy plus comcomitant and adjuvant temozolomide for glioblastoma. N Engl J Med 2005; 352: 987-96.

2. Fitzek, M.M., Thornton, A.F., Rabinov, J.D., et al. Accelerated fractionated proton/photon irradiation to 90 cobalt gray equivalent for glioblastoma multiforme: results of a phase II prospective trial. J Neurosurg. 1999; 91: 251-260.

3. Tanaka M, Ino $\mathrm{Y}$, Nakagawa $\mathrm{k}$, et al. High-dose conformal radiotherapy for supratentorial malignant glioma: a historical comparison. Lancet Oncol. 2005; 6: 953-960.

4. Walker MD, Green SB, Byar DP, et al. Randomized comparisons of radiotherapy and nitrosoureas for the treatment of malignant glioma after surgery. $\mathrm{N}$ Engl J Med 1980; 303: 1323-1329.

5. Kristiansen K, Hagen S, Kollevold T, et al. Combined modality therapy of operated astrocytomas grade III and IV: Confirmation of the value of postoperative irradiation 
and lack of potentiation of bleomycin on survival time: A prospective multicenter trial of the Scandinavian Glioblastoma Study Group. Cancer 1981; 47: 649-652.

6. Sandberg-Wollheim M, Malmstrom P, Stromblad LG, et al. A randomized study of chemotherapy with procarbazine, vincristine, and the lomustine with and without radiation therapy for astrocytoma grade 3 and/or 4. Cancer 1991; 68: 22-29.

7. Bleehen NM, Stennning SP. A Medical Research Council trial of two radiotherapy doses in the treatment of grades 3 and 4 astrocytoma. Br J Cancer 1991; 64: 769-774.

8. Nwokedi EC, DiBase SJ, Jabbour S, et al. Gamma knife stereotactic radiosurgery for patients with glioblastoma multiforme. Neurosurgery 2002; 50: 41-47.

9. Baumert BG, Lutterbach J, Bernays R, et al. Fractionated stereotactic radiotherapy boost after post-operative radiotherapy in patients with high-grade gliomas.

Radiotherapy and Oncology 2003; 67: 183-190.

10. Souhami L, Seiferheld W, Brachman D, et al. Randomized comparison of stereotactic radiosurgery followed by conventional radiotherapy with carmustine to conventional radiotherapy with carmustine for patients with glioblastoma multiforme: report of Radiation Therapy Oncology Group 93-05 protocol. Int J Radiat Oncol Biol Phys. 2004; 60: 853-860.

11. Yamamoto T, Matsumura A, Nakai K, et al. Current Clinical Results of the Tsukuba BNCT Trial. Applied Radiation Isotopes 2004; 61: 1089-1093.

12. Miyatake S, Kawabata S, Kajimoto Y, et al. Modified boron neutron capture therapy for malignant gliomas performed using epithermal neutron and two boron compounds with different accumulation mechanisms: an efficacy study based on findings on neuroimages. J Neurosurg2005; 103:1000-1009.

13. Chanana AD, Capala J, Chadha M, et al. Boron neutron capture therapy for glioblastoma multiforme: Interim results from the phase I/II dose-escalation studies. Neurosurgery 1999; 44: 1182-1193.

14. Kageji T, Nagahiro S, Matsuzaki K, et al. Boron neutron capture therapy using mixed epithermal and thermal neutron beams in patients with malignant glioma-correlation between radiation dose and radiation injury and clinical outcome. Int. J. Radiat. Oncol. Biol. Phys. 2006; 65: 1446-1455.

15. Stenstam BH, Pellettieri L, Sorteberg W, et al. BNCT for recurrent intracranial meningeal tumours - case reports. Acta Neurol Scand 2007; 115: 243-7.

16. Busse PM, Harling OK, Palmer MR, et al. A critical examination of the results from the Harvard-MIT NCT program phase I clinical trial of neutron capture therapy for intracranial disease. Journal of Neuro-Oncology 2003; 62: 111-121.

17. Barth RF, Coderre JA, Vicente GH, et al. Boron Neutron Capture Therapy of Cancer: 
Current Status and Future Prospects. Clin Cancer Res 2005; 11: 3987-4002.

18. Capala, J.H., Stenstam, B.H., Sköld, K., et al. Boron neutron capture therapy for glioblastoma multiforme. Journal of Neuro-Oncology 2003; 62: 135-144.

19. Diaz, A.Z. Assessment of the results from the phase I/II boron neutron capture therapy trials at the Brookhaven National Laboratory from a clinician's point of viewJournal of Neuro-Oncology 2003; 62: 101-109.

20. Coderre JA, Turcotte JC, Riley KJ, et al. Boron neutron capture therapy: Cellular targeting of high linear energy transfer radiation. Technol Cancer Res T 2003; 2: 1-21.

21. Witig, A., Hideghety, K., Paquis, P., et al. Current clinical results of the EORTC-study 11961. In: Surewein W, Moss R, Wittig A, editors. Research and Development in Neutron Capture Therapy. Bologna: Monduzzi Editore; 2002. p.1117-22

22. Joensuu, H., Kankaanranta, L., Seppälä, T., et al. Boron neutron capture therapy of brain tumors: clinical trials at the Finnish Facility using boronophenylalanine. J Neurooncol $2003 ; 62: 123-134$.

23. Burian, J., Marek, M., Rataj, J., et al. Report on the first group of the phase I BNCT trial at the LVR-15 reactor. In: Surewein W, Moss R, Wittig A, editors. Research and Development in Neutron Capture Therapy. Bologna: Monduzzi Editore; 2002. p.1107-1112.

24. Aihara T, Hiratsuka J, Morita N, et al. First clinical case of boron neutron capture therapy for head and neck malignancies using 18F-BPA PET. Head Neck 2006; 28 : 850-855.

25. Mishima, Y. Melanoma and non-melanoma neutron capture therapy using gene therapy: Overview. In: Larsson B, Crawford J, Weinreich R. Advances in Neutron Capture Therapy. Medicine and Physics, Excerpta Medica ICS 1132, vol. I. Amsterdam: Elsevier; 1997. pp. 10-25

26. González SJ, Bonomi MR, Santa Cruz GA, et al. First BNCT treatment of a skin melanoma in Argentina: dosimetric analysis and clinical outcome. Appl Radiat Isot. 2004; 61: 1101-1105.

27. Wittig A, Malago M, Collette L, et al. Uptake of two ${ }^{10} \mathrm{~B}$-compounds in liver metastases of colorectal adenocarcinoma for extracorporeal irradiation with boron neutron capture therapy (EORTC Trial 11001). Int J Cancer 2008; 122: 1164-71.

28. Suzuki M, Sakurai Y, Hagiwara S, et al. First attempt of boron neutron capture therapy (BNCT) for hepatocellular carcinoma. Jpn J Clin Oncol. 2007; 37: 376-81.

29. Kankaanranta L, Seppälä T, Koivunoro H, et al. Boron neutron capture therapy in the treatment of locally recurred head and neck cancer. Int J Radiat Oncol Biol Phys 2007; 69: 475-82. 
30. Yamamoto T, Matsumura A, Yamamoto K, et al. Characterization of neutron beams for boron neutroncapture therapy: in-air radiobiological dosimetry. Radiat.Res. 2003; 160: $70-76$.

31. Curran WJ, Scott CB, Horton J, et al. Recursive partitioning analysis of prognostic factors in three Radiation Therapy Oncology Group Malignant Glioma Trials, J Natl Cancer Inst 1993; 85: 704-710.

32. Mirimanoff RO, Gorlia T, Mason W, et al. Radiotherapy and temozolomide for newly diagnosed glioblastoma: Recursive partitioning analysis of the EORTC 26981/22981-NCIC CE3 phase III randomized trial. J Clin Oncol 2006; 24: 2563-2569.

33. Trotti A, Byhardt R, Stetz J, et al. Common toxicity criteria: version 2.0. an improved reference for grading the acute effects of cancer treatment: impact on radiotherapy. Int J Radiat Oncol Biol Phys 2000; 47: 13-47.

34. Shrieve DC, Eben A, Black PM, et al. Treatment of patients with primary glioblastoma multiforme with standard postoperative radiotherapy and radiosurgical boost:

Prognostic factors and long-term outcome. J Neurosurg 1999; 90: 72-77.

35. Nakagawa Y, Pooh K, Kobayashi T, et al. Clinical review of the Japanese experience with boron neutron capture therapy and a proposed strategy using epithermal neutron beams. J Neurooncol; 2003: 62, 87-99.

36. Barth R.F., Grecula, J.C., Yang, W. et al. Combination of boron neutron capture therapy and external beam radiotherapy for brain tumors. Int. J. Radiation Oncology Biol. Phys. 2004; 58: 267-277.

37. Soloway, A.H., Hatanaka, H., Davis, M.A. Penetration of brain and brain tumor. VII. Tumor-binding sulfhydryl boron compounds. J. Med. Chem. 1967; 10: 714-747.

38. Hideghety K, Sauerwein W, Wittig A, Gotz C, Paquis P, Grochulla F, Haselsberger K, Wolbers J, Moss R, Huiskamp R, Fankhauser H, de Vries M, Gabel D. Tissue uptake of BSH in patients with glioblastoma in the EORTC 11961 phase I BNCT trial. J Neurooncol. 2003; 62: 145-56.

39. Yoshida, F., Matsumura, A., Shibata, Y., Yamamoto, T., Nakauchi, H., Okumura, M., Nose, T. Cell cycle dependence of boron uptake from two boron compounds used for clinical neutron capture therapy. Cancer letters 2002; 187: 135-141.

40. Joel DD, Coderre JA, Micca PL, Nawrocky MM. Effect of dose and infusion time on the delivery of p-boronophenylalanine for neutron capture therapy. J Neurooncol. 1999; 41: 213-221.

41. Smith D, Chandra S, Barth R, et al. Quantitative imaging and microlocalization of boron-10 in brain tumors and infiltrating tumor cells by SIMS ionmicroscopy: 
42. Barth RF, Jensuu H. Boron neutron capture therapy for the treatment of glioblastomas and extracranial tumours: As effective, more effective or less effective than photon irradiation? Radiotherapy and Oncology 2007; 82: 119-122. 